\title{
Effect of Forced Cooling Contraction on Shrinkage Cavity and Segregation of Ultra-thick Continuous Casting Slab
}

\author{
Jiazheng ZHANG, Ling YAN, Changjun XU*, Dejun LI*, Lianwang ZHANG
}

\begin{abstract}
Due to the shrinkage cavities and macro segregation that frequently happened in ultra-thick slab and affected the slab quality, a new technique called forced cooling contraction, which added extra cooling water in solidification end has been provided. The slice moving boundary model was established, the secondary cooling zone was divided into rolling zone, continuous bending zone and segment arc zone. The accuracy of secondary cooling calculation model was verified. The influence on shrinkage cavities and macro segregation was researched when the water flow was from 1,5;2,5; 4 and 7 times of original water flow. Compared with original water flow, when time of water flow in segment arc zone is lower than 2,5 , the shrinkage cavities length decreases. When water flow is 2,5 times of original water flow, the segregation degree and ratio decreased from 1,325 and 0,963 to 1,294 and 0,930 , respectively. Combine with the results of shrinkage cavities and macro segregation. The considerable strength of forced cooling is 2,5 times of original water flow.
\end{abstract}

Keywords: forced cooling contraction; macro segregation; shrinkage cavities; ultra-thick slab

\section{INTRODUCTION}

The ultra-thick slab is widely used in shipbuilding, military industry and nuclear facilities. The traditional method of making ultra-thick slab is die casting, but this method has low productivity and high energy costs. Due to the high demand for ultra-thick slab, the production of ultra-thick slab has been mostly replaced by continuous casting [1]. In recent years, researchers found the size of ultra-thick slab is increasing [2-5], which is shown in Tab. 1.

Table 1 The size of slab around the world

\begin{tabular}{|c|c|}
\hline Group Company & Size of ultra-thick slab \\
\hline $\begin{array}{c}\text { Pohang Iron and Steel Co. Ltd, } \\
\text { Korea }\end{array}$ & $250 \sim 400 \times 1000 \sim 2000 \mathrm{~mm}$ \\
\hline Nagoya, Japan & $4002360 \mathrm{~mm}$ \\
\hline Dillingen, Germany & $600 \mathrm{~mm}$ thickness \\
\hline Ansteel Co., Ltd. China & $250 \sim 300 \mathrm{~mm}$ thickness \\
\hline $\begin{array}{c}\text { Nanyang Hanye Special Steel Co., } \\
\text { Ltd. China }\end{array}$ & $420 \times 2700 \mathrm{~mm}$ \\
\hline
\end{tabular}

Compared with thin slab, size below $100 \mathrm{~mm}$, continuous casting, ultra-thick slab continuous casting has lower casting speed, higher latent heat and longer liquid core [6]. During casting process, shrinkage cavities and macro segregation usually happened due to unreasonable solidifying.

Researches show that changing the water distribution of secondary cooling zone will control shrinkage cavities and macro segregation [7-9]. The influence of spraying intensity on solidification structure was studied by Shili Zhu. Zhu concluded that when the water flow is approached, the dendrite spacing of thick slab is higher than that in thin slab. The strong cooling should be used to reduce the space between dendrites of thick slab, controlling the generation to the macro segregation. Pengli Dong et al. agree that using strong cooling technique will make the crystallization rapidly, which controls the macro segregation. However, Dong said that if secondary cooling is too strong, the speed of solidification will increase, the casting feeding will not be in time. The shrinkage cavities will be provided in slab center. With many steel plants using gradually decreased secondary cooling water while manufacturing ultra-thick slab, the quality in solidification end cannot be guaranteed.

Forced cooling contraction is a new method by adding extra cooling water in solidification end. The suitable forced cooling water can increase the speed of solidification appropriately. The temperature gradient at solidification front will increase; the shrinkage cavities can be controlled. The strong water flow will also add extra contraction stress to boundary, which will decrease expansion stress provided by latent heat release, the suction effects of expansion are reduced, and the macro segregation will be controlled.

In this study, the simulation is taken by ProCAST. The effect of forced cooling contraction of solidification end on shrinkage cavities and macro segregation, and the feasibility of this method using in steel plant is estimated. The suggested water flow of forced cooling contraction is also provided.

\section{PARAMETERS OF MODEL}

In this paper, physical and mathematical modeling is established for the heat transfer from the slab to secondary cooling water.

\subsection{Material and Casting Parameters}

X70 has been used as an experiment material. The content of elements is shown in Tab. 2. The liquidus and solidus are $1782 \mathrm{~K}$ and $1702 \mathrm{~K}$, respectively.

\begin{tabular}{|c|c|c|c|c|c|c|c|c|}
\hline Element & C & $\mathrm{Mn}$ & Mo & $\mathrm{P}$ & $\mathrm{S}$ & $\mathrm{Si}$ & $\mathrm{Ti}$ & V \\
\hline Content & 0,16 & 1,70 & 0,05 & 0,02 & 0,01 & 0,45 & 0,06 & 0,06 \\
\hline
\end{tabular}

Ultra-thick slab was produced by Vertical Bending Continuous Caster; the parameters of caster are shown in Tab. 3 .

The secondary cooling zone has been divided into 8 regions with 16 segments. According to the function of each zone, the secondary cooling zone has been simplified into four zones: rolling zone, continuous bending zone, and segment arc zone, see in Fig. 1. The forced cooling 
contraction will be put in segment arc zone (from 1 to 15 ). The water distribution of each zone is shown in Tab. 4.

Table 3 Parameters of vertical bending continuous caster
\begin{tabular}{|c|c|}
\hline Items & Value \\
\hline The Effective Length of Mold & $0,8 \mathrm{~m}$ \\
\hline Temperature Difference of Mold Cooling Water & $4,5 \mathrm{~K}$ \\
\hline Total Length of Continuous Casting & $33,09 \mathrm{~m}$ \\
\hline The Specific Heat of Water & $4200 \mathrm{Jkg}^{-1} \mathrm{~K}^{-1}$ \\
\hline Superheat & $30 \mathrm{~K}$ \\
\hline Casting Speed & $0,8 \mathrm{mmin}^{-1}$ \\
\hline Totally Secondary Cooling Water Flow & $188,9 \mathrm{Lmin}^{-1}$ \\
\hline
\end{tabular}

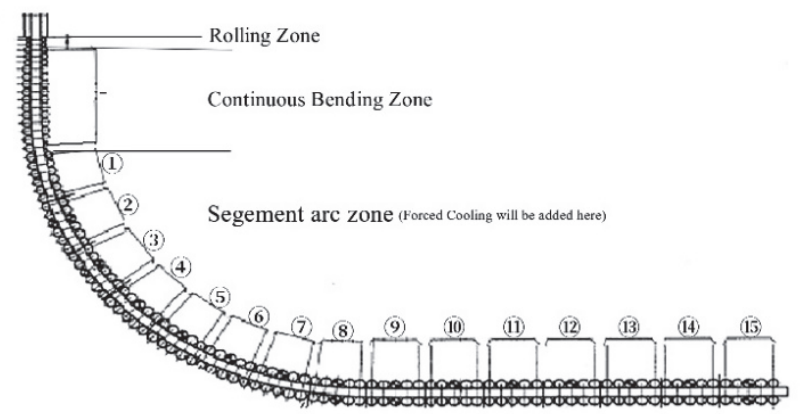

Figure 1 Location of secondary cooling zone

Table 4 Water distribution ratio of secondary cooling zone

\begin{tabular}{|c|c|c|c|}
\hline Zones & Rolling zone & $\begin{array}{c}\text { Continuous } \\
\text { Bending Zone }\end{array}$ & $\begin{array}{c}\text { Segment arc } \\
\text { zone }\end{array}$ \\
\hline $\begin{array}{c}\text { Water } \\
\text { Distribution Ratio } \\
\zeta_{i}\end{array}$ & 0,27 & 0,33 & 0,4 \\
\hline
\end{tabular}

where $\xi_{i}$ is the water distribution ratio of each secondary cooling zone. The coefficients of rolling zone, continuous bending zone and segment arc zone are $\xi_{1}, \xi_{2}, \xi_{3}$, respectively.

\subsection{Assumptions and Mesh Generation \\ 2.2.1 Assumptions of Calculation Mode}

In the simulation process, the heat taken in by the model is equal to the heat released. The model heat transfer has been concluded by Eq. (1).

$$
\rho C \frac{\partial T}{\partial t}=\frac{\partial}{\partial x}\left(\theta \frac{\partial T}{\partial x}\right)+\frac{\partial}{\partial y}\left(\theta \frac{\partial T}{\partial y}\right)+\frac{\partial}{\partial z}\left(\theta \frac{\partial T}{\partial z}\right)+Q_{l}
$$

where $C$ is specific heat, $\mathrm{Jkg}^{-1} \mathrm{~K}^{-1} . T$ is temperature, $\mathrm{K} ; t$ is solidification time, s; $Q_{l}$ is latent heat, J. $\rho C(\partial T / \partial t)$ is heat change caused by temperature or mass flow, J. $\partial[\theta(\partial T / \partial x)] / \partial x, \quad \partial[\theta(\partial T / \partial y)] / \partial y, \quad \partial[\theta(\partial T / \partial z)] / \partial z$ are the components of the heat flow in the $x, y, z$ direction, respectively.

In order to simplify the simulation, the following assumptions have been carried out [10].

(1) The heat release of molten steel can be totally taken away by cooling water. wall.

(2) There are no air gaps between mold and copper

(3) The heat transfer between slab and roller is ignored.

(4) The water quantity of each zone is regarded as constant

because the slice moving boundary model (slice model) can be regarded as a slice with no thickness at the transient state. By the assumptions above, the 2-D non-steady model can be regarded as 2-D steady model.

\subsubsection{The Generation of Mesh and Settings}

In this study, the slice model has been used. The size of the slice model is as follows: $230 \mathrm{~mm}$ long, $1950 \mathrm{~mm}$ wide and $20 \mathrm{~mm}$ thick. By using mesh options in ProCAST, 16742 2-D elements and 48117 3-D elements have been created. In simulation, mold boundary condition is added in side surface. Secondary cooling and forced cooling conditions are added in width direction. The mesh and boundary condition zones of model are shown in Fig. 2, where $\mathrm{BC}$ is boundary conditions.

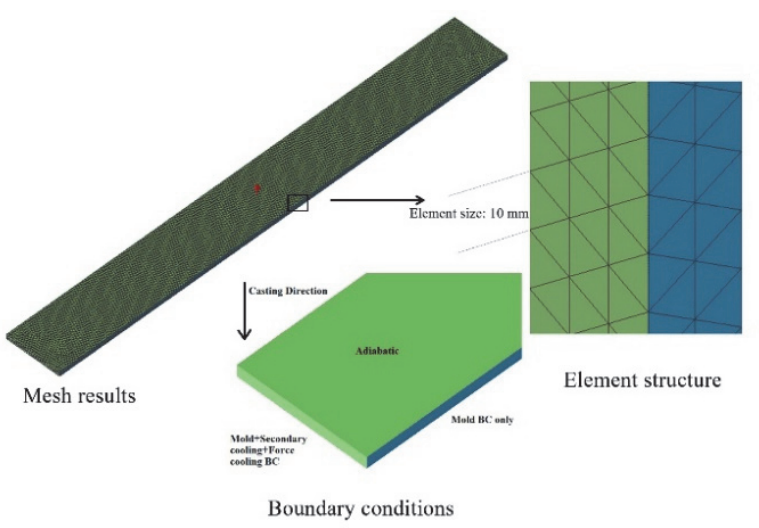

Figure 2 The mesh and boundary condition zones of model

\subsection{Thermal Boundary Conditions}

The inner temperature, $T_{0}(\mathrm{~K})$ is equal to casting temperature, $T_{\mathrm{C}}(\mathrm{K})$ at the beginning of simulation, i.e.,

$T_{0}=T_{\mathrm{c}}$

The heat transfer of mold can be expressed as:

$q_{\text {mold }}=\frac{Q_{\mathrm{W}} C_{\mathrm{W}} \Delta T_{\mathrm{W}}}{S_{\mathrm{B}}}$

where, $Q_{\mathrm{W}}$ is total water flow in mold; $C_{\mathrm{W}}$ is specific heat of water, $\mathrm{Jkg}^{-1} \mathrm{~K}^{-1} ; \Delta T_{\mathrm{W}}$ is temperature differences of mold cooling water, $\mathrm{K} ; S_{\mathrm{B}}$ is the attached area between molten steel and copper wall, $\mathrm{m}^{2}$.

The film coefficient of secondary cooling zone is connected with intensity of water distribution, which can be concluded as Eq. (4).

$h_{\mathrm{sec}}=a W^{b}+c$

where, $h_{\mathrm{sec}}$ is film coefficient in secondary cooling. $a, b, c$ are constants, which can be confirmed by experiment results; $W$ is the intensity of water distribution, $\mathrm{Lm}^{-2} \mathrm{~s}^{-1}$. By establishing a relationship with the total water volume, density, section size and drawing speed of the secondary cooling zone, Eq. (4) can be changed as: 


$$
h_{\mathrm{sec}}^{\prime}=a\left[\frac{\xi\left(W_{0} \rho S_{1} v+m\right)}{s_{0}}\right]^{b}+c
$$

where $m$ is correction factor of film coefficient. $Q_{\text {sec }}$ is total water flow of secondary cooling zone, $\mathrm{Lmin}^{-1} ; s_{0}$ is the area of spray water $\mathrm{m}^{2} . \rho, S_{1}, v$ are destiny $\left(\mathrm{kgm}^{-3}\right)$, totally lateral area $\left(\mathrm{m}^{2}\right)$ and casting speed $\left(\mathrm{mmin}^{-1}\right)$, respectively.

The heat transfer of radiation zone can be expressed as Eq. (6).

$$
q_{\mathrm{rad}}=\varepsilon \sigma\left[T_{\mathrm{b}}^{4}-T_{\mathrm{out}}^{4}\right]
$$

where, $q_{\text {rad }}$ is film coefficient of radiation zone, $\mathrm{Wm}^{-2} ; \varepsilon$ is blackness, selected as $0.8, \sigma$ is constant of Stephen Boltzmann, which is $5.67 \times 10^{-8} ; T_{\text {out }}$ is the external temperature, K. $T_{\mathrm{b}}$ is the boundary temperature, $\mathrm{K}$.

\subsection{Shrinkage Cavities Prediction Model}

Niyama model is frequently used as a prediction model of shrinkage cavities. Niyama shrinkage criterion is provided by Niyama in Japan [11]. The distribution of shrinkage cavities in different sections and compositions of casting slabs has been analyzed by Niyama. Eq. (7) shows the solution equation of Niyama.

$$
N y=\frac{G}{\sqrt{R}}
$$

where the unit of $N y$ is $\mathrm{Cmin}^{-1 / 2} \mathrm{~cm}^{-1 / 2}, G$ and $R$ is temperature gradient $\mathrm{Ccm}^{-1}$ and solidification rate, $\mathrm{cmmin}^{-1}$, respectively. When Niyama is smaller than 1 , the shrinkage cavities of slab will happen.

\subsection{Macro-segregation Mode}

The equation of Clyne-Kurz [12] was used to calculate macro segregation, which is shown in Eq. (8) to Eq. (10).

$C_{\mathrm{L}}=C_{0}\left[1-(1-2 \alpha k) f_{\mathrm{s}}\right]^{\frac{k-1}{1-2 \alpha k}}$

$\alpha=\frac{4 D_{s} t_{f}}{\lambda^{2}}$

$t_{\mathrm{f}}=\frac{T_{\mathrm{L}}-T_{\mathrm{S}}}{C_{\mathrm{R}}}$

where, $k$ is the equilibrium distribution coefficient of solid and liquid phase in solidification front. $C_{0}$ is initial concentration of solution in molten steel; $f_{\mathrm{s}}$ is fraction solid of slab; $D_{\mathrm{s}}$ diffusion coefficient in solid. $\lambda$ is secondary arm spacing; $T_{\mathrm{L}}$ and $T_{\mathrm{S}}$ are liquidus temperature and solidus temperature, respectively. $C_{\mathrm{R}}$ is solidification rate.

When analyzing the segregation data, the segregation degree and segregation rate are usually used to measure the segregation level. The Equation is shown in Eq. (11) and Eq. (12).
$S_{\mathrm{e}}=\frac{C_{\min }}{C_{\max }}$

$S_{\mathrm{R}}=\frac{C_{\mathrm{max}}-C_{\min }}{C_{\mathrm{ave}}}$

where, $C_{\max }, C_{\min }, C_{\text {ave }}$ is the maximum, minimum and average content of Carbon in center-line direction, respectively.

\section{ANALYSIS AND DISCUSSION \\ 3.1 The Accuracy of Calculation Model}

In this part, the accuracy of secondary cooling calculation model is verified by comparing the experiment and simulation result of liquid core length. Nail-shooting experiment is often used to estimate the liquid core length. The nail-shooting facility is combined with nail with sulfur groove and shooting equipment. When the nail was shot in slab, sulfur groove in nail will melt in molten steel instead of solidification shell [13]. After solidification of slab, the sulfur groove in nail represents the thickness of solidification shell. The solidification square root law is shown in Eq. (13).

$D=K \sqrt{t}=K \sqrt{l / v}$

where, $D$ is shell thickness, $\mathrm{mm} ; K$ is combined heat transfer coefficient, mmmin $^{-1 / 2} ; t$ is solidification time, $\mathrm{s} ; l$ is the distance to the meniscus, $\mathrm{m}$.

The equation of liquid core length has been changed into Eq. (14).

$l=\frac{D^{2} v}{K^{2}}$

In order to simplify the simulation steps, $b$ is selected as 0,6 , and $c$ is $0, m$ in each zone is $0 ; 393$ and 550, respectively. The simulation results by changing the value of a, in Eq. (5), have been shown in Fig. 3. The composite solidification coefficient of three groups is shown in Tab. 5 .

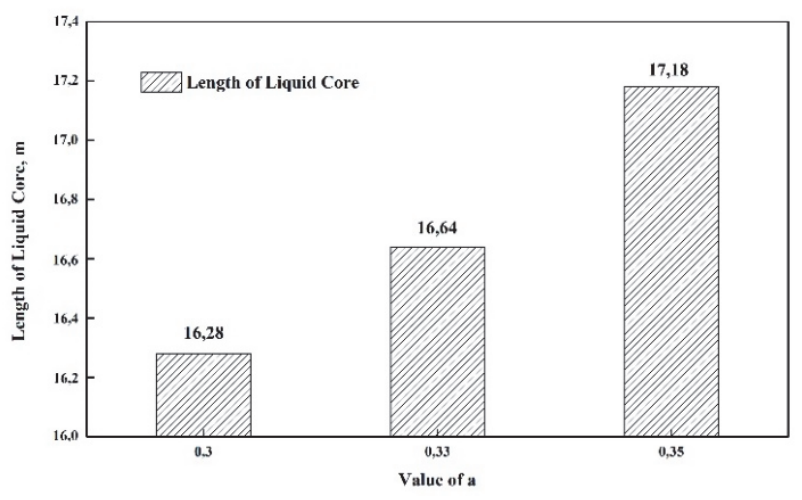

Figure 3 Fraction solid in different value of a

It can be concluded from Fig. 3 and Tab. 5, when $a$ is 0,33 , the liquid core length of slab is $16,64 \mathrm{~m}$, the composite solidification coefficient is 25,21. The 
experiment composite solidification coefficient is 25,24 . The coefficient is similar to experiment results, so the accuracy of secondary cooling calculation model is evaluated.

Table 5 Composite solidification coefficient in different a

\begin{tabular}{|c|c|c|}
\hline$a$ & $\begin{array}{c}\text { Composite Solidification } \\
\text { coefficient, } \text { mmmin }^{-1 / 2}\end{array}$ & $\begin{array}{c}\text { Liquid Core Estimated } \\
\text { by Nail-shooting } \\
\text { Experiment }\end{array}$ \\
\hline 0,3 & 24,81 & \multirow{2}{*}{25,24} \\
\hline 0,33 & 25,21 & \\
\hline 0,35 & 25,62 & \\
\hline
\end{tabular}

The calculation equation of each part in secondary cooling zone is expressed as shown in Tab. 6 .

Table 6 Calculation equation of each part in secondary cooling zone

\begin{tabular}{|c|c|}
\hline $\begin{array}{c}\text { Secondary } \\
\text { cooling zone }\end{array}$ & Calculation Equation \\
\hline Rolling zone & $h_{\mathrm{sec} 0}^{\prime}=0,33\left(\xi_{0} \frac{235,23 v}{s_{0}}\right)^{0,6}$ \\
\hline $\begin{array}{c}\text { Continuous } \\
\text { Bending Zone }\end{array}$ & $h_{\mathrm{sec} 1}^{\prime}=0,33\left[\frac{\xi_{1}(235,23 v+393)}{s_{0}}\right]^{0,6}$ \\
\hline $\begin{array}{c}\text { Segment Arc } \\
\text { Zone }\end{array}$ & $h_{\mathrm{sec} 2}^{\prime}=0,33\left[\frac{\xi_{2}(235,23 v+550)}{s_{0}}\right]^{0,6}$ \\
\hline
\end{tabular}

In the present work, the boundary conditions have been determined. The following contents will affect the shrinkage cavities and segregation of the slab under different forced cooling strength. Finally, the most suitable forced shrinkage cooling conditions will be selected.

\subsection{Influences on Shrinkage Cavities}

The original cooling is a standard water flow in steel plant. Different strength of forced cooling is provided by different times of original cooling, see in Tab. 7.

Table 7 The strength of forced cooling

\begin{tabular}{|c|c|c|}
\hline $\begin{array}{c}\text { Forced cooling } \\
\text { strength }\end{array}$ & $\begin{array}{c}\text { Water flow of } \\
\text { segment arc zone } \\
/ \text { Lmin }^{-1}\end{array}$ & $\begin{array}{c}\text { Times of original } \\
\text { cooling }\end{array}$ \\
\hline Original cooling & 75,27 & 1,0 \\
\hline Weak cooling & 112,91 & 1,5 \\
\hline Medium cooling & 188,19 & 2,5 \\
\hline Mid-strong cooling & 301,10 & 4,0 \\
\hline Strong cooling & 526,89 & 7,0 \\
\hline
\end{tabular}

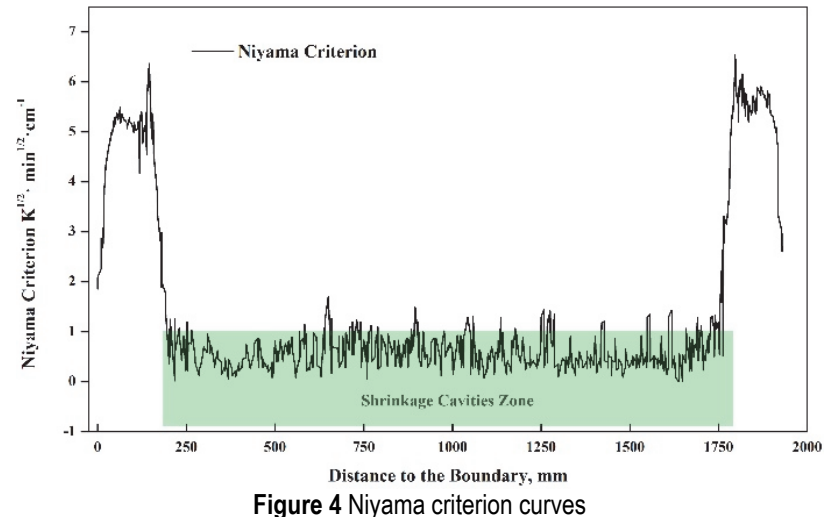

Thermal simulation has been taken by ProCAST. In wide surface, the Niyama criterion in center-line direction of ultra-thick slab has been provided in Fig. 4. The green zone is the shrinkage cavities zone. The length of shrinkage cavities zone in each group was obtained, which has been shown in Fig. 5.

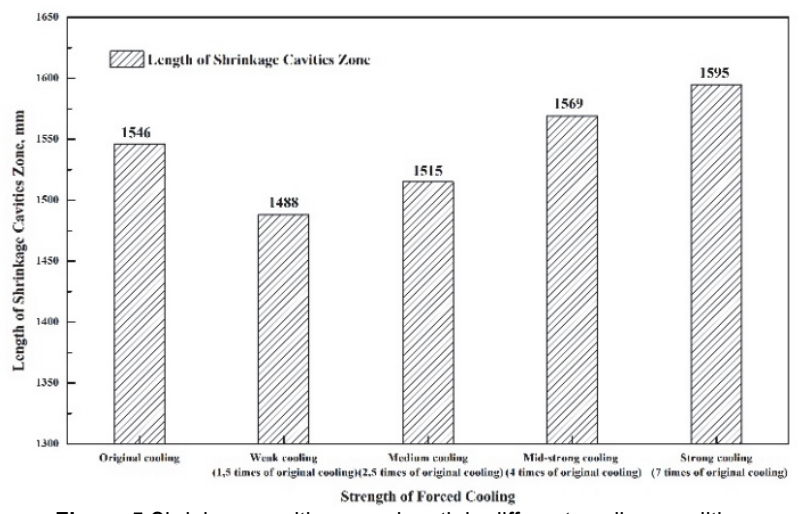

Figure 5 Shrinkage cavities zone length in different cooling condition

According to Fig. 5, the length of shrinkage cavities decreases then increases with the increasing strength of force cooling contraction. When the water flow is 1,5 times of original cooling, the shrinkage cavity zone is $1488 \mathrm{~mm}$, while the value is $1546 \mathrm{~mm}$ in original cooling. With the water flow of solidification end increasing, the length of shrinkage cavities zone rises to $1595 \mathrm{~mm}$, which is not useful for controlling shrinkage cavities.

The shrinkage cavities zone in narrow surface is also discussed. However, both secondary cooling and the force cooling are added in width direction. The influence of shrinkage cavities in narrow surface is small. The length remained around $25 \mathrm{~mm}$.

In conclusion, the shrinkage cavities can be controlled in a weak extra cooling in solidification end. The strength of forced cooling is suggested no more than 2,5 times of original water flow. According to opinions of Pengli Dong [9], in the following part, the influence on macro segregation has been also discussed in order to find a cooling that can control macro segregation.

\subsection{Influence on Macro-segregation}

The content of $\mathrm{C}$ is regarded as a research object in this study. The distribution of $\mathrm{C}$ at center line of wide surface is shown in Fig. 6. It can be concluded that positive segregation will happen in slab center-line. The red zone in the figure shows the positive segregation area in slab.

c Overall |pwt:
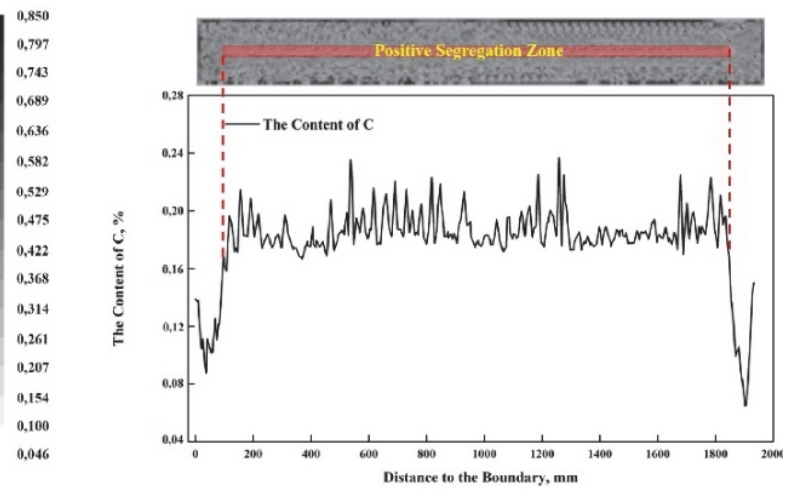

Figure 6 The content of $C$ and the location of positive segregation zone 
The original cooling, the weak, medium, mid-strong and strong forced cooling are also investigated. The macro segregation simulation results are shown in Tab. 8. It can be concluded that using forced cooling contraction at the end of solidification end can mitigate the average content of $\mathrm{C}$ both in wide surface and positive segregation zone. It means that the fluctuation of carbon content in the direction of the center line will be reduced. The macro segregation will be controlled.

Table 8 Static data of macro segregation parameter

\begin{tabular}{|c|c|c|}
\hline $\begin{array}{c}\text { Forced cooling } \\
\text { strength }\end{array}$ & $\begin{array}{c}\text { Average content of C } \\
\text { in positive segregation } \\
\text { zone }\end{array}$ & $\begin{array}{c}\text { Average content of C } \\
\text { in slab center-line of } \\
\text { wide surface }\end{array}$ \\
\hline Original cooling & 0,1789 & 0,1863 \\
\hline Weak cooling & 0,1782 & 0,1858 \\
\hline Medium cooling & 0,1783 & 0,1859 \\
\hline Mid-strong cooling & 0,1784 & 0,1860 \\
\hline Strong cooling & 0,1786 & 0,1862 \\
\hline
\end{tabular}

According to Eq. (11) and Eq. (12), the segregation degree and segregation rate in center-line direction have been shown in Fig. 7. with the increasing forced cooling strength. The segregation degree and segregation rate first decrease then increase. The maximum value of segregation degree and rate is 1,329 and 0,965 with the 1,5 times of ordinary cooling. The minimum value of segregation degree and rate is 1,294 and 0,930 with the 2,5 times of ordinary cooling. Results show that using forced cooling construction can control segregation.

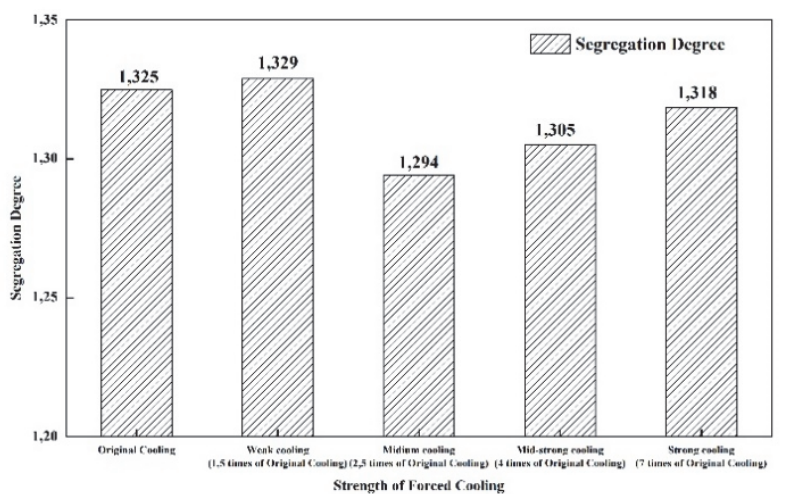

(a) Segregation degree

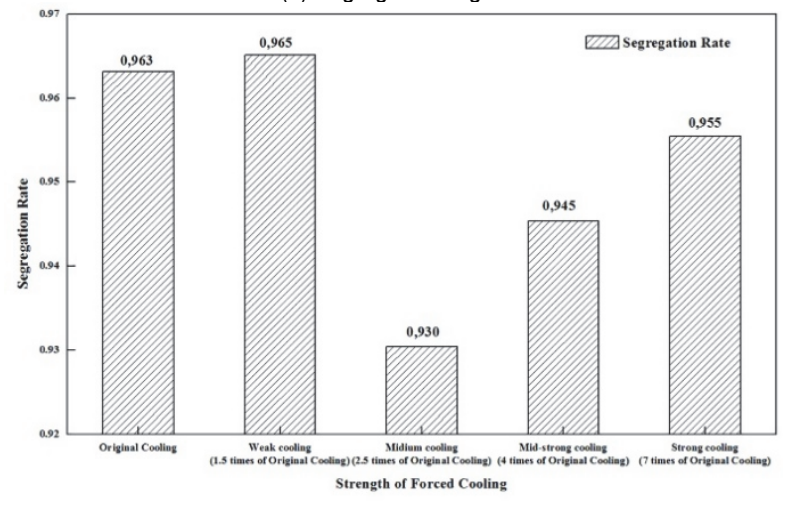

(b) Segregation rate

Figure 7 Segregation degree and rate in different strength of forced cooling

Combined with simulation and statistic data of shrinkage cavities zone length and macro segregation, when the water flow is more than 1,5 times but no more than 2,5 times, both the shrinkage cavities and macro segregation will be controlled. In production practice, 2,5 times of ordinary cooling is better to select as forced cooling strength, because both of the macro segregation and shrinkage cavities have been controlled, and improve the quality of slabs.

\section{CONCLUSION}

In this paper, the slice moving boundary model was used and the secondary cooling calculation model was verified by nail-shooting experiment. The extra water flow was added to segment arc zone. The influence on shrinkage cavities and macro segregation of different strength of forced cooling was researched. The appropriate force cooling water flow was chosen. According to the research, the following conclusions are reached:

(1) When the multiple water flow of segment arc zone increases from 1 to 7 . The length of shrinkage cavities in center-line direction first decreases then increases. The shrinkage cavities can be controlled in a weak extra cooling in solidification end. The strength of forced cooling is suggested no more than 2,5 times of original water flow, which can reduce shrinkage cavity zone length.

(2) The influence on macro segregation is also investigated, When the strength of forced cooling is no more than 2,5 times, the segregation degree decreases from 1,325 to 1,294 , and the segregation ratio decreases from 0,963 to 0,930 . The degree and ratio increases when the water flow rises continuously.

(3) Combined with the results of shrinkage cavities and macro segregation. The forced cooling contraction method is feasible. The considerable strength of forced cooling is 2,5 times of original water flow.

\section{Acknowledgments}

The authors are grateful for the financial support from the National Natural Science Foundation of China (No. 51504130) and Joint Fund of HGSKL-USTLN (No. HGSKL-USTLN(2020)02). Partial support was also provided by Special Fund of USTL (No. 2017HZ01) and USTL Talent Project (No. 601011507-15).

\section{REFERENCES}

[1] Xie, X., Chen, D., Long, M., Zhang, L., Shen, J., \& Youguang M. (2013). Numerical Analysis of Coupled Fluid Flow, Heat Transfer and Solidification in Ultra-thick Slab Continuous Casting Mold. Materials Processing Fundamentals, 63-71. https://doi.org/10.1007/978-3-319-48197-5_7

[2] Wang, Z., Zhao, J., Wang, Y., Zhang, H., Zhen, X., \& Hu, X. (2019). Research on Central Segregation Control of $400 \mathrm{~mm}$ ultra-thick slab. Continuous Casting, 44(6), 47-50. https://doi.org/10.13228/j.boyuan.issn1005-4006.20190017

[3] Wang X., Xu, S., Zhao, D., \& Qiao, H. (2013). The Causes and Control Measures for Longitudinal Surface Cracks on $400 \times 2100 \mathrm{~mm}$ Specially Thick and Wide Steel Plates. Continuous Casting, 2, 35-37. https://doi.org/10.13228/j.boyuan.issn10054006.2013.02.009

[4] Xiong, W., Yu, X., Yu, J., Xie, G., Wang, H., \& Pei, W. (2017). Application of Large Reduction of $420 \mathrm{~mm}$ Ultra-thick Slabs. Henan Metallurgy, 25(2), 39-42.

[5] Cui, F., Sun, W., Zhao, Q., \& Yu, X. (2013). Development of Manufacturing Technology of Heavy-gauge Steel Plate in China. Shandong Metallurgy, 35(1), 1-6. 
https://doi.org/10.16727/j.cnki.issn1004-4620.2013.01.028

[6] Liu, S., Ai, S., Long, M., Wu, S., Xu, P., Chen, D., \& Wang, Q. (2019). Numerical Simulation of Heat Transfer between Roll and Slab under Dry Secondary Cooling in Ultra-thick Slab Continuous Casting. Steel Research International, 91(3).

[7] Pierer, R. \& Bernhard, C. (2008). On the Influence of Carbon on Secondary Dendrite Arm Spacing in Steel. Journal of Materials and Science, 43(21), 6938-6943. https://doi.org/10.1007/s10853-008-2985-3

[8] Zhu S., Zheng, G., Ye J., Hu, W., \& Cao, H. (2014). Influence of Spraying Intensity on Solidification Structure of Continuous Casting Billet during Secondary Cooling. Iron and Steel, 49(8), 48-53. https://doi.org/10.13228/j.boyuan.issn0449-749x.20130542

[9] Dong, P., Shang, H., \& Wang, H. (2017). Typical Quality Defect Cause and Control Technology of Slab and Plate. China Metallurgy, 27(6), 7-13. https://doi.org/10.13228/j.boyuan.issn1006-9356.20160239

[10] Ueshima, Y, Mizoguchi, S., Matsumiya, T., \& Kajioka, H. (1986). Analysis of Solute Distribution in Dendrites of Carbon Steel with $\delta / \gamma$ Transformation during Solidification. Metallugical. Transaction. B,17, 845-859. https://doi.org/10.1007/BF02657148

[11] Lee P., Chirazi, A., \& See, D. (2001). Modeling Microporosity in Aluminum-silicon Alloys: a review. Journal of Light Metals. 1(1), 15-30. https://doi.org/10.1016/s1471-5317(00)00003-1

[12] Clyne, T. W. \& Kurz, W. (1981). Solute redistribution during solidification with rapid solid state diffusion. Metallurgical Transactions A, 12(6), 965-971. https://doi.org/10.1007/bf02643477

[13] Hou A., Min Y., Liu, C., \& Jiang, M. (2015). Heat Transfer and Solidification Model of Slab Continuous Casting Based on Nail-Shooting Experiments. Advanced Materials Research, 1088, 153-158. https://doi.org/10.4028/www.scientific.net/AMR.1088.153

\section{Contact information:}

Jia-zheng ZHANG, Postgraduate

School of Materials and Metallurgy,

University of Science and Technology Liaoning,

Anshan, People's Republic of China

State Key Laboratory of Metal Material for Marine Equipment and Application

E-mail: kapok1a96@163.com

Chang-jun XU, PhD, Assistant Professor

(Corresponding author)

School of Materials and Metallurgy,

University of Science and Technology Liaoning,

Anshan, People's Republic of China.

E-mail: jassonas@163.com

Ling YAN, Senior engineer

State Key Laboratory of Metal Material for Marine Equipment and Application, Anshan, Liaoning Province, People's Republic of China

E-mail: geqeg801ycnc2@163.com

Dejun LI, Senior engineer

(Corresponding author)

State Key Laboratory of Metal Material for Marine Equipment and Application, Anshan, Liaoning Province, People's Republic of China.

E-mail: 416177019@qq.com

\section{Lian-wang ZHANG, PhD}

School of Materials and Metallurgy,

University of Science and Technology Liaoning,

Anshan, People's Republic of China

E-mail: zhanglianwang92@163.com 